\title{
Electronic charge rearrangement at metal/organic interfaces induced by weak van der Waals interactions
}

\author{
Nicola Ferri, ${ }^{1}$ Alberto Ambrosetti, ${ }^{2}$ and Alexandre Tkatchenko ${ }^{1,3}$ \\ ${ }^{1}$ Fritz-Haber-Institut der Max-Planck-Gesellschaft, Faradayweg 4-6, 14195, Berlin, Germany \\ ${ }^{2}$ Dipartimento di Fisica e Astronomia, Università degli Studi di Padova, 35131 Padova, Italy \\ ${ }^{3}$ Physics and Materials Science Research Unit, University of Luxembourg, L-1511 Luxembourg
}

(Received 3 May 2017; revised manuscript received 14 June 2017; published 25 July 2017)

\begin{abstract}
Electronic charge rearrangements at interfaces between organic molecules and solid surfaces play a key role in a wide range of applications in catalysis, light-emitting diodes, single-molecule junctions, molecular sensors and switches, and photovoltaics. It is common to utilize electrostatics and Pauli pushback to control the interface electronic properties, while the ubiquitous van der Waals ( $\mathrm{vdW}$ ) interactions are often considered to have a negligible direct contribution (beyond the obvious structural relaxation). Here, we apply a fully self-consistent Tkatchenko-Scheffler vdW density functional to demonstrate that the weak vdW interactions can induce sizable charge rearrangements at hybrid metal/organic systems (HMOS). The complex vdW correlation potential smears out the interfacial electronic density, thereby reducing the charge transfer in HMOS, changes the interface work functions by up to $0.2 \mathrm{eV}$, and increases the interface dipole moment by up to 0.3 Debye. Our results suggest that $\mathrm{vdW}$ interactions should be considered as an additional control parameter in the design of hybrid interfaces with the desired electronic properties.
\end{abstract}

DOI: 10.1103/PhysRevMaterials.1.026003

\section{INTRODUCTION}

Interfaces between materials with distinct properties may exhibit unexpected phenomena, resulting in novel interface functionalities that are not possessed by either of the materials separately. In this regard, nanoscale interfaces are especially appealing because of quantum-size effects that emerge in such materials. In particular, hybrid metal/organic systems (HMOS) are used as models for novel interfaces due to the high electrical conductivity of the metal and the tunable structural, electronic, and optical properties of organic molecules [1,2]. For example, HMOS composed of anode-cathode sandwiches or thin films of organic molecules on metal substrates show significant promise for applications as components for transistors, lightemitting diodes, switches, sensors, and photovoltaics $[1,3,4]$.

The adsorption of molecules on a metal surface implies a complex rearrangement of the electron density $(\Delta n)$, connected to the formation of intrinsic dipoles and, consequently, to interfacial electrostatic interactions. Therefore, the electronic properties of HMOS, as the band gap or the work function $(\Phi)$, can be modulated by controlling the electrostatics at the interface. This feature opens the possibility to explore new functionalities in organic electronic devices [5] and to improve their performances $[3,6]$.

Density functional theory (DFT) is the preferred theoretical method to model electronic properties of extended interfaces (hundreds of atoms) because of its computational efficiency. In this context, the long-range van der Waals ( $\mathrm{vdW}$ ) interactions - while absent in standard DFT exchangecorrelation (XC) functionals-have been proven of fundamental importance in determining the structure and the cohesive energies of layered systems, such as molecules adsorbed on metal surfaces [3,7-14]. On the other hand, the long-range $\mathrm{vdW}$ energy represents only a small fraction $(0.001 \%)$ of the total electronic energy for a wide range of systems [15], from small dimers (e.g., diatomic dimers, water dimer) to complex hybrid inorganic/organic systems, passing through large molecular systems and metal surfaces. Hence, the influence of $\mathrm{vdW}$ interactions on the electronic properties is typically assumed to be rather small, if not negligible. For this reason, vdW effects are usually incorporated a posteriori, i.e., as a perturbative correction of the total DFT energy. However, doing so amounts to an uncontrolled approximation.

In this work we investigate the role of $\mathrm{vdW}$ interactions on the electronic properties of HMOS via a fully self-consistent (SC) implementation of the Tkatchenko-Scheffler $\mathrm{vdW}^{\text {surf }}$ functional [12]. Previous electronic structure calculations indicate that vdW interactions can affect the electronic properties of extended systems, such as metallic surfaces [15]. Moreover, the intermolecular $\mathrm{vdW}$ interactions are found to play a prominent role in improving the performances of molecular diodes [3]. However, an exhaustive and detailed study on the specific role of long-range $\mathrm{vdW}$ interactions on the electronic structure and properties of organic/inorganic systems is still absent to date.

The findings presented herein indicate that the long-range correlation effects entail unexpectedly large electron density rearrangements. Notably, vdW interactions can contribute up to $20 \%-50 \%$ of the total $\Delta n$, induced by molecular adsorption. Consequently, the electrostatics of the hybrid systems are modified, resulting in changes of the interfacial dipoles of $0.2-0.3 \mathrm{D}$. In addition to that, our results show that the long-range correlation interactions are responsible for a substantial reduction of the transfer of charge between the substrate and the monolayer. In this context, an experimentally accessible property is the shift of the work function of the metal surface upon molecular adsorption $(\Delta \Phi)$. The combination of the vdW-induced effects listed above is reflected into nontrivial modifications of the work-function shifts on the order of $\sim 0.20 \mathrm{eV}$. For the systems presented in the following these large modifications can correspond to one-third or even half of the total $\Delta \Phi$. Most importantly, the self-consistent treatment of $\mathrm{vdW}$ interactions is found to systematically improve the 
agreement between the theoretical prediction of $\Delta \Phi$ and the experimentally determined data.

The general validity of our findings is assessed by considering several HMOS of different size and chemical compositions. Moreover, surfaces with different Miller indices are also taken into account. The selected cases are representative of the various substrate-monolayer interactions, spanning from model weakly interacting systems to complex interfaces. The detailed analysis presented here provides insights into the fundamental contributions of $\mathrm{vdW}$ interactions for correctly predicting and controlling the electronic properties of HMOS. As such, this work is strongly indicative of the potential importance of self-consistency also for the properties of hybrid inorganic-semiconductor/organic systems. Finally, our results suggest that the long-range correlation interactions could yield nontrivial effects in properties beyond the ground state.

\section{MOTIVATION AND PREVIOUS WORK}

Tuning the electronic properties of HMOS is an outstanding challenge, investigated both theoretically [13,16-19] and experimentally [20-23]. For example, the shift of the metal work function upon molecular adsorption, one of the most important properties of HMOS, can be achieved by three distinct effects: (i) the Pauli pushback, i.e., the repulsion between the electrons belonging to the metal surface and those of the adsorbed molecules; (ii) the presence of a charge transfer between the surface and the monolayer; (iii) the intrinsic electronic dipole of the monolayer. The first two phenomena produce electrostatic dipoles located at the interface and are also responsible for the work-function pinning effect [24]. Consequently, the goal of carefully tailoring the electrostatics at the interface can be achieved by (indirectly) manipulating these three factors.

In this regard, much progress has been made using a variety of different techniques, from doping methods [25,26] to charge carrier injector/acceptor layers [27-29] and stacking layers [20,30]. In general, a chemisorbed monolayer with a pronounced polar orientation is usually associated with a large $\Delta \Phi$, on the order of $2-3 \mathrm{eV}$ [31]. On the other hand, weakly bonded interfaces, as in the case of physisorbed molecules on metals, display a modest shift of about $0.5 \mathrm{eV}$ [32]. However, HMOS often present the coexistence of different molecules, molecular orientations, lateral interactions, etc. For such complex interfaces, the work-function shift exhibits a nontrivial relationship with complex structural modifications. For instance, stacking additional monolayers, although visibly modifies the geometry of the HMOS, can result in modest $\Delta \Phi$ of about $0.1 \mathrm{eV}$ [30].

From a theoretical point of view, the correct prediction of the geometry is a fundamental prerequisite in order to characterize the electronic structure of HMOS. In past years, several methods emerged for including the key contribution of vdW interactions into the DFT framework [33-35]. Among all remedies, few SC implementations are available. For example, the SC vdW-DF [36] has been employed with layered systems as melamine, naphthalenetetracarboxylic dianhydride (NTCDA) and perylenetetracarboxylic dianhydride (PTCDA) on a gold (111) surface [7]. For such extended and complex systems, SC calculations revealed that vdW interactions yield substantial electron density rearrangements. Notably, they can be responsible for about half of the total displacement of electron density, induced upon molecular adsorption [7]. However, the SC vdW-DF produces overestimated work functions for clean metal surfaces [15]. On the other hand, the $\Phi$ values computed with the $\mathrm{SC}$ vdW ${ }^{\text {surf }}$ functional are in very good agreement with experimental data, with particularly large effects for $\mathrm{Cu}, \mathrm{Rh}$, and $\mathrm{Ag}$ (111) surfaces [15]. Therefore, in this work we employ the vdW ${ }^{\text {surf }}$ functional and its SC version. In the following we introduce the theoretical methodology in more detail. Then, the role of vdW interactions in the electronic structure of hybrid systems is illustrated with a case-by-case analysis.

\section{SELF-CONSISTENT TKATCHENKO-SCHEFFLER VAN DER WAALS DENSITY FUNCTIONAL}

The vdW functional utilized in this work is the extension of the pairwise Tkatchenko-Scheffler (TS) functional [34], which enables the modeling of the adsorption of atoms and molecules on surfaces [12] $\left(\mathrm{vdW}^{\text {surf }}\right)$. This scheme accounts for the collective many-body response of the metal substrate via a renormalization of the vdW parameters. In actual DFT calculations, the vdW contributions are usually computed a posteriori. In this way the computational cost of the vdW functional is negligible with respect to the whole DFT calculation. As a drawback, the vdW effects on the electron density are not considered. As mentioned above, this approximation is motivated by the fact that the $\mathrm{vdW}$ term is responsible only for a small part of the total energy.

On the other hand, the vdW effects on the electronic structure are achieved only within a self-consistent scheme. In this case, the main ingredient is the long-range correlation potential, obtained by deriving the energy expression of the functional with respect to the electron density. The potential for $\mathrm{vdW}^{\text {surf }}$ is

$$
v_{\mathrm{vdW} \text { surf }}[n]=-\frac{1}{2} \sum_{A B} \frac{\delta}{\delta n}\left(f_{A B}[n] \frac{C_{6, A B}[n]}{R_{A B}^{6}}\right),
$$

where the dependency on the position is omitted to simplify the notation. The application of the chain rule in Eq. (1) gives the derivative of two terms and both depend on $n(\mathbf{r})$. Finally, the vdW potential is included, as a part of the XC potential, into the Hamiltonian: $v[n]=v_{\mathrm{xc}}[n]+v_{\mathrm{vdW} \text { surf }}[n]$. Thus, the ground-state $n(\mathbf{r})$ is affected by $\mathrm{vdW}$ interactions in a seamless way.

The SC implementation presented in this work has been optimized, in the absence of any cutoff, to treat extended systems with hundreds of atoms. Considering the largest cases tested up to date-HMOS that contain about 200-300 metal atoms and organic molecules - the cost of a fully self-consistent calculation, with tight basis sets, is roughly $20 \%-30 \%$ of a standard DFT calculation.

We have used the full-potential all-electron code FHI-AIMS [37] for all the DFT results presented here. The calculations with vdW effects are obtained employing both the a posteriori $\left(\mathrm{vdW}^{\text {surf }}\right)$ and the SC ( $\left.\mathrm{vdW}_{\mathrm{sc}}^{\text {surf }}\right)$ implementations, in combination with the $\mathrm{XC}$ functional of Perdew, Burke, and Ernzerhof [38] (PBE). 
TABLE I. Summary of binding distances, binding energies, and work-function shifts for CuPc, benzene, DIP, and PTCDA adsorbed on $\mathrm{Ag}(111)$. The results listed here are obtained with $\mathrm{PBE}+\mathrm{vdW}$ surf, $\mathrm{PBE}+\mathrm{vdW}_{\mathrm{sc}}^{\text {surf }}$, and from experiments. The average distances between carbon $\left(d_{\mathrm{C}}\right)$, nitrogen $\left(d_{\mathrm{N}}\right)$, and copper $\left(d_{\mathrm{Cu}}\right)$ atoms and the topmost metal layer are reported in angstroms. The binding energies $E_{b}$ and the work-function shifts $\Delta \Phi$ are in electronvolts.

\begin{tabular}{|c|c|c|c|c|c|c|c|c|c|c|c|c|c|c|}
\hline & \multicolumn{5}{|c|}{$\mathrm{CuPc}$} & \multicolumn{3}{|c|}{ Benzene } & \multicolumn{3}{|c|}{ DIP } & \multicolumn{3}{|c|}{ PTCDA } \\
\hline & $d_{\mathrm{C}}$ & $d_{\mathrm{N}}$ & $d_{\mathrm{Cu}}$ & $E_{b}$ & $\Delta \Phi$ & $d_{\mathrm{C}}$ & $E_{b}$ & $\Delta \Phi$ & $d_{\mathrm{C}}$ & $E_{b}$ & $\Delta \Phi$ & $d_{\mathrm{C}}$ & $E_{b}$ & $\Delta \Phi$ \\
\hline $\mathrm{PBE}+\mathrm{vdW}$ surf & 3.00 & 2.99 & 2.87 & -4.085 & -0.22 & $2.96^{\mathrm{c}}$ & -0.74 & -0.72 & $3.00^{\mathrm{g}}$ & -3.22 & -0.18 & $2.84^{\mathrm{i}}$ & -3.06 & 0.33 \\
\hline $\mathrm{PBE}+\mathrm{vdW}$ sc & 3.00 & 2.99 & 2.87 & -4.012 & -0.41 & 2.96 & -0.73 & -0.86 & 3.00 & -3.18 & -0.39 & 2.84 & -3.00 & 0.11 \\
\hline Experiment & $3.08^{\mathrm{a}}$ & $3.04^{\mathrm{a}}$ & $2.97^{\mathrm{a}}$ & & $-0.44^{\mathrm{b}}$ & $3.04^{\mathrm{d}}$ & $-0.69^{\mathrm{e}}$ & $-0.94^{\mathrm{f}}$ & $3.01^{\mathrm{g}}$ & & $-0.44^{\mathrm{h}}$ & $2.86^{\mathrm{j}}$ & & $0.06^{\mathrm{k}}-0.16^{\mathrm{l}}$ \\
\hline
\end{tabular}

${ }^{\mathrm{a}}$ Reference [39]; ${ }^{\mathrm{b}}$ Reference [30]; ${ }^{\mathrm{c}}$ Reference [9]; ${ }^{\mathrm{d}}$ Reference [40]; ${ }^{\mathrm{e}}$ Reference [41]; ${ }^{\mathrm{f}}$ Reference [42]; ${ }^{\mathrm{g}}$ Reference [11]; ${ }^{\mathrm{h}}$ Reference [43]; ${ }^{\mathrm{i}}$ Reference [12]; ${ }^{\mathrm{j}}$ Reference [44]; ${ }^{\mathrm{k}}$ Reference [45]; ${ }^{\mathrm{l}}$ Reference [46].

\section{COMPLEX CHARGE REARRANGEMENT: COPPER PHTHALOCYANINE ON SILVER}

As a first example of HMOS, we present an aromatic molecule of the family of metal-phthalocyanines (MePc): CuPc adsorbed on $\operatorname{Ag}(111)$ and $\operatorname{Ag}(100)$ surfaces. The main characteristic of this class of organic semiconductors is the presence of a metal atom in the center of the molecule. Depending on the metal atom, the molecular geometry assumes a planar or nonplanar configuration and displays different electronic and magnetic properties, e.g., the vertical dipole. The possibility of a controlled and efficient property tuning by just replacing the metal atom, combined with a good thermal stability, make this class of molecules of particular interest for applications in organic electronics, such as in photovoltaic cells, sensors, and LED. CuPc on coinage metal surfaces has been characterized with several experimental techniques, such as as scanning tunneling microscopy (STM), x-ray standing wave (XSW), and ultraviolet photoemission spectroscopy (UPS) $[39,47]$. Recently, this molecule has been utilized in a multicomponent blend with perfluoropentacene (PFP) in order to study the modification of the interfacial properties with respect to the single-component system [30]. Nevertheless, these systems are particularly challenging for standard DFT functionals. In fact, the metal-molecule interfaces are weakly bounded and the vdW dispersion interactions are of primary importance for a correct description of the geometries [14].

Before the analysis of the electronic structure and properties, the system was relaxed using the $\mathrm{PBE}+\mathrm{vdW}$ surf functional. The computational details and the definitions of adsorption energy and distance can be found in the Supplemental Material [48]. For the well-characterized $\mathrm{CuPc} / \mathrm{Ag}(111)$, the binding distance between the topmost metal layer and the carbon atoms $\left(d_{\mathrm{C}}\right)$ is reported in Table I, along with $d_{\mathrm{Cu}}$ and $d_{\mathrm{N}}$. The three results show very good agreement with the experimental measurements, with the largest discrepancy being $0.1 \AA$ for $d_{\mathrm{Cu}}$. The binding energy of this system, computed with $\mathrm{PBE}+\mathrm{vdW}$ surf , is $-4.08 \mathrm{eV}$. In this regard, the inclusion of self-consistency, via the $\mathrm{PBE}+\mathrm{vdW}_{\mathrm{sc}}^{\text {surf }}$ method, yields a small change of $2 \%$ in the binding energy.

The next step is an in-depth analysis of the vdW effects on $n(\mathbf{r})$. We define the electron density modification induced by molecular adsorption as $\Delta n(\mathbf{r})=n(\mathbf{r})_{\mathrm{HMOS}}-\left[n(\mathbf{r})_{\text {surf }}+\right.$ $\left.n(\mathbf{r})_{\text {mol }}\right]$, where the electron densities of the two isolated fragments are subtracted to that of the whole system. Before proceeding, we remark here that the $\mathrm{PBE}+\mathrm{vdW^{ \text {surf } }}$ functional consists of a vdW correction of the total energy. Therefore, the electron density of this functional corresponds to that of $\mathrm{PBE}$, referred to as $\Delta n(\mathbf{r})_{\mathrm{PBE}}$. After computing $\Delta n(\mathbf{r})$, using both PBE and PBE $+\mathrm{vdW}_{\mathrm{sc}}^{\text {surf }}$, we considered the difference between the two induced electron densities, $\Delta n(\mathbf{r})_{\mathrm{vdW}}=$ $\Delta n(\mathbf{r})_{\mathrm{PBE}+\mathrm{vdW}}^{\text {surf }}-\Delta n(\mathbf{r})_{\mathrm{PBE}}$. A two-dimensional (2D) cross section of $\Delta n(\mathbf{r})_{\mathrm{vdW}}$ is plotted in Fig. 1. The slice corresponds to an $x-y$ plane located between the topmost metal layer and the molecular plane. From the figure it emerges that, when vdW contributions are included self-consistently, the electron density displays collective, pronounced, and complex redistributions at the organic/metal interface, which is the region of primary importance for the electronic properties of HMOS. In the particular case of $\mathrm{CuPc} / \operatorname{Ag}(111)$, the depletion regions overlaps with the footprint of the molecule. The accumulation areas are found at the copper metal atom and around the molecule, see Fig. 1(a). Such large density redistributions imply a modification of the electrostatics at the interface and, consequently, of the work function.

The work function is defined as the minimum energy required to remove an electron from the bulk to a point in the vacuum:

$$
\Phi=V_{\text {vacuum }}-E_{F},
$$

where $V_{\text {vacuum }}$ is the electrostatic potential in the vacuum and $E_{F}$ the Fermi energy. The work function of the metal surface displays a shift $\Delta \Phi$ upon molecular adsorption. In the specific case of $\mathrm{CuPc} / \operatorname{Ag}(111)$, vdW interactions induce a periodic array of permanent and macroscopic dipoles at the interface, leading to a shift $\Delta \Phi=-0.41 \mathrm{eV}$, to be compared to an experimental result of $-0.44 \mathrm{eV}$, see Table I. On the contrary, when SC vdW effects are absent, PBE alone captures only half of this shift $(-0.22 \mathrm{eV})$. Similar results are found with $\mathrm{CuPc} / \mathrm{Ag}(100)$. Namely, the binding energies of $\mathrm{PBE}+\mathrm{vdW}$ surf and $\mathrm{PBE}+\mathrm{vdW}_{\mathrm{sc}}^{\text {surf }}$ show a small discrepancy $(1.5 \%)$, while $\Delta \Phi$ increases from $-0.14 \mathrm{eV}$ to $-0.22 \mathrm{eV}$ when SC vdW effects are included. However, the rearrangement of $n(\mathbf{r})$ is qualitatively different with respect to $\mathrm{CuPc} / \mathrm{Ag}(111)$. For example, $\mathrm{CuPc} / \mathrm{Ag}(100)$ shows an accumulation of density at the $\mathrm{Cu}$ atom, see Fig. 1(b). These differences in the density are a consequence of the different adsorption sites. In fact, $\mathrm{CuPc} / \mathrm{Ag}(100)$ adsorbs in a top site, while a hollow site is preferred for $\mathrm{CuPc} / \mathrm{Ag}(111)$, and the electron density is accumulated around the copper atom of the molecule. 
(a)

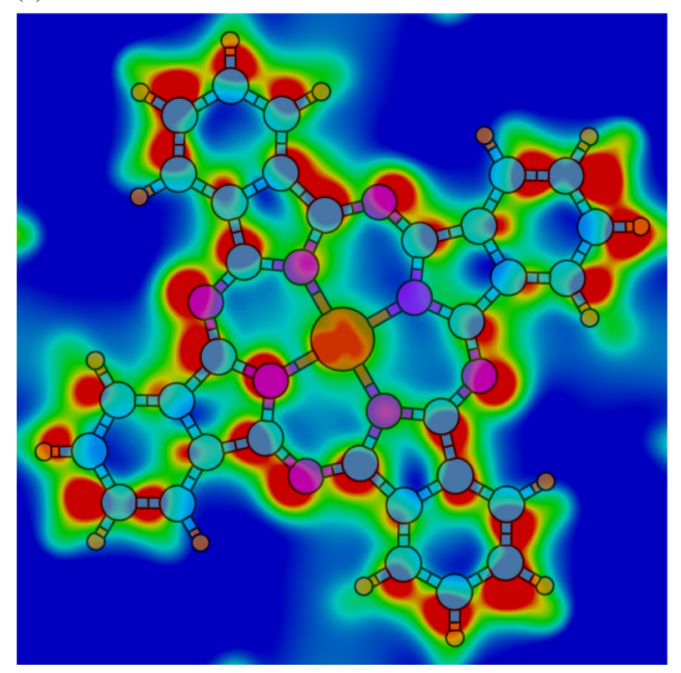

(b)

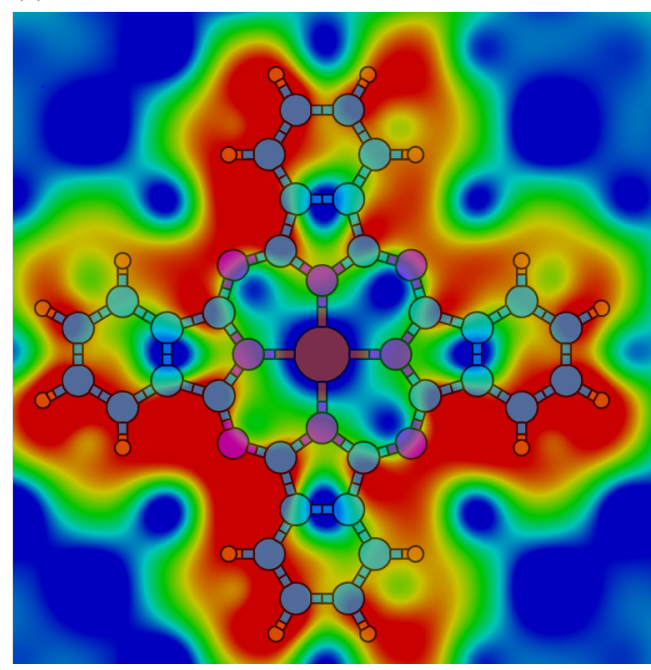

FIG. 1. 2D slices of vdW-induced electron density [ $\left.\Delta n(\mathbf{r})_{\mathrm{vdW}}\right]$. The regions of electron density accumulation are in blue, while red indicates depletion. (a) Top view of CuPc on $\mathrm{Ag}(111)$. The limiting values are set to $\pm 5.0 \times 10^{-4} \mathrm{e} / \AA^{3}$. (b) Top view of CuPc on $\mathrm{Ag}(100)$. The limiting values are set to $\pm 4.0 \times 10^{-4} \mathrm{e} / \AA^{3}$. The CuPc molecule is sketched as a guide to the eye. In both figures the inclusion of vdW interactions produce large electron density rearrangements. The differences in the accumulation/depletion regions between the two isosurfaces stem from the fact that the molecule adsorbs with different configurations, depending on the type of surface employed.

\section{FROM SIMPLE TO COMPLEX INTERFACES}

To gain insights into the effects of self-consistency, three additional HMOS of increasing complexity (and size) are considered. As a first example, we start with a small molecule: benzene (Bz). This molecule has been highly used and studied as an adsorbate on $\operatorname{Ag}(111)$ [42,49,50] and is considered a model system for larger hydrocarbons. Here, $\mathrm{Bz}$ is adsorbed in a flat configuration [49] at a distance of $2.96 \AA$ [9]. The binding energies, obtained with both $\mathrm{PBE}+\mathrm{vdW}^{\text {surf }}$ and $\mathrm{PBE}+\mathrm{vdW}_{\mathrm{sc}}^{\text {surf }}$ (Table I), indicate a rather weak physisorption. Therefore, this HMOS represents a particularly useful example for our analysis inasmuch as the charge transfer is practically absent. The flat configuration of $\mathrm{Bz}$ prevents the formation of a molecular dipole. Thus, the only phenomenon involved in the modification of the interface dipole is the Pauli pushback effect [51]. The result is an accumulation of electron density close to the metal surface, which yields a dipole that points towards the surface. The metal work function is reduced accordingly. In this regard, the inclusion of vdW interactions produces a delocalization of electron density above the silver surface and around $\pi$-conjugated molecules [15]. This enhances the overlap between electronic wave functions and triggers a larger Pauli pushback effect. Therefore, a dipole of $1.22 \mathrm{D}$ is found with $\mathrm{PBE}$, while $\mathrm{SC}$ vdW increases the dipole to $1.46 \mathrm{D}$. Consequently, the $\triangle \Phi$ of PBE increases by $0.14 \mathrm{eV}$ $(\sim 20 \%)$ when $\mathrm{vdW}$ interactions are included, reaching an agreement between theory and experiment to better than $0.1 \mathrm{eV}$.

The analysis and the results presented above can be expanded by considering another well-characterized HMOS: diindenoperylene (DIP, $\mathrm{C}_{32} \mathrm{H}_{16}$ ) on $\mathrm{Ag}(111)$. DIP is a $\pi$ conjugated semiconductor and has a relatively simple chemical structure: it is a planar hydrocarbon. This molecule has excellent optoelectronic device performances [52,53]. DIP has been studied extensively in monolayer on coinage metal surfaces
[54-56]. The comparison between the equilibrium distance $d_{C}$ obtained with PBE $+v d^{\text {surf }}$ and XSW experiments (Table I) shows an excellent agreement [11]. For what concerns $E_{b}$, self-consistency leads to a tiny reduction $(1.25 \%)$ of the $\mathrm{PBE}+\mathrm{vdW}^{\text {surf }}$ value. From the point of view of the electronic properties, a charge transfer is present between the DIP molecule and the metallic surface. In this HMOS, the flow of charge, from the substrate to the molecule, fills the unoccupied molecular orbitals. As a consequence, an interface dipole which points away from the surface is established. The charge transfer effectively counterbalances the Pauli pushback repulsion, increasing the metal $\Phi$. Nevertheless, in the specific case of DIP/ $\operatorname{Ag}(111)$, the pillow effect remains the predominant factor and the resulting $\Delta \Phi$ is still negative. Self-consistency produces here a $\Delta \Phi$ value of $-0.39 \mathrm{eV}$, doubling the PBE $+\mathrm{vdW}^{\text {surf }}$ value and significantly improving the agreement with the experimental result (Table I).

We consider now a 2D slice of $\Delta n(\mathbf{r})_{\mathrm{vdW}}$, taken along the path of the dotted line in Fig. 2(a). From this 2D section, visualized in Fig. 2(b), it is evident that self-consistency predicts a depletion of charge at the molecular monolayer. Notably, the electron density depletion extends over the whole plane of the molecule. In fact, the volume of the depletion regions around the molecule is much larger than the molecule itself and the 2D plane cuts the electron density depletion of five rings (see Supplemental Material [48]). Conversely, for what concerns the metal surface, there is a density accumulation between the silver metal layers and in the vacuum region, while a depletion is found at the metal atoms [15]. A quantitative inspection of these effects is provided in Fig. 2(c), by considering $\Delta n(\mathbf{r})_{\mathrm{vdW}}$ averaged over the $x-y$ plane and plotted as a function of $z$, the axis perpendicular to the metal surface. In this figure, a dipolelike density redistribution emerges. One pole is located at the interface (positive), the other is close to the monolayer (negative). Having examined the electron density, we now 

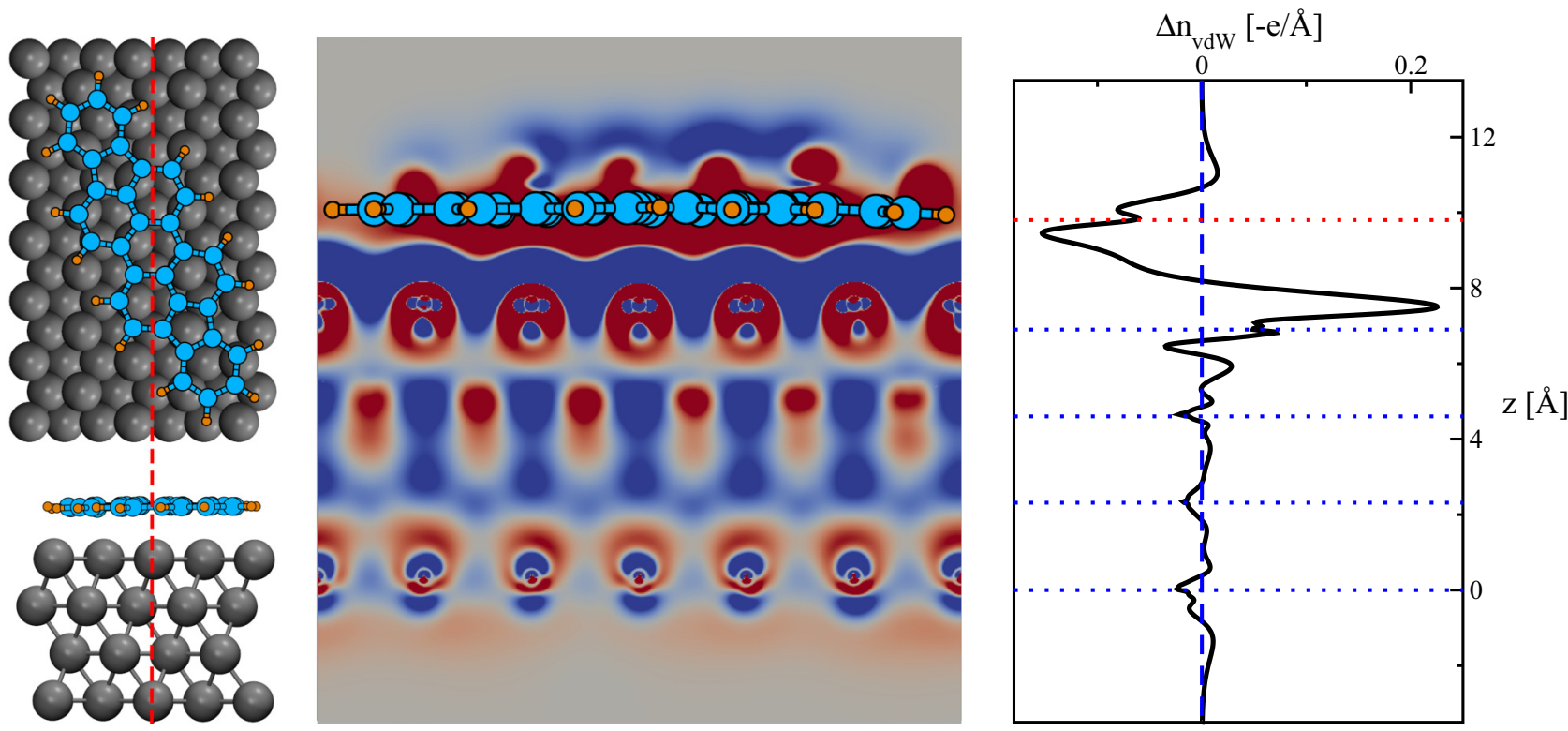

FIG. 2. DIP on $\operatorname{Ag}(111)$. Left: Top and side view of the unit cell, the red dashed line indicates the 2D plane used to cut a $2 \mathrm{D}$ slice of $\mathrm{vdW}$-induced electron density [ $\Delta n(\mathbf{r})_{\mathrm{vdW}}$ ]. Center: The $2 \mathrm{D}$ isosurface of $\Delta n(\mathbf{r})_{\mathrm{vdW}}$ displays the vdW effect on the electron density distribution, accumulation is in blue, depletion is in red. The limiting values are set to $\pm 2.0 \times 10^{-4} \mathrm{e} / \AA^{3}$. The profile of the DIP molecule is sketched as a guide to the eye. Right: The integral of $\Delta n(\mathbf{r})_{\mathrm{vdW}}$ is plotted as a function of $z$, the axis perpendicular to the surface. A dipolelike density redistribution emerges at the interface. The horizontal dotted lines indicate the monolayer plane and the four metal layers; this plot is aligned with the figure shown in the central panel.

compute the total displaced charge, $Q(z)$, i.e., the integral of the density difference $\Delta n(z)$ (see Supplemental Material [48]). In Fig. 3 are plotted the $Q(z)$ associated with PBE and $\mathrm{PBE}+\mathrm{vdW}$ sc . Large differences are visible at the interface region. Overall, $\mathrm{SC}$ vdW increases the total displaced charge by about $0.2 \mathrm{e}$. In detail, the positive peak located just above the topmost metal layer, which denotes the Pauli pushback effect, is roughly $40 \%$ higher with SC effects. Furthermore, the downward slope, located between the substrate and the monolayer, indicates the presence of a charge transfer directed

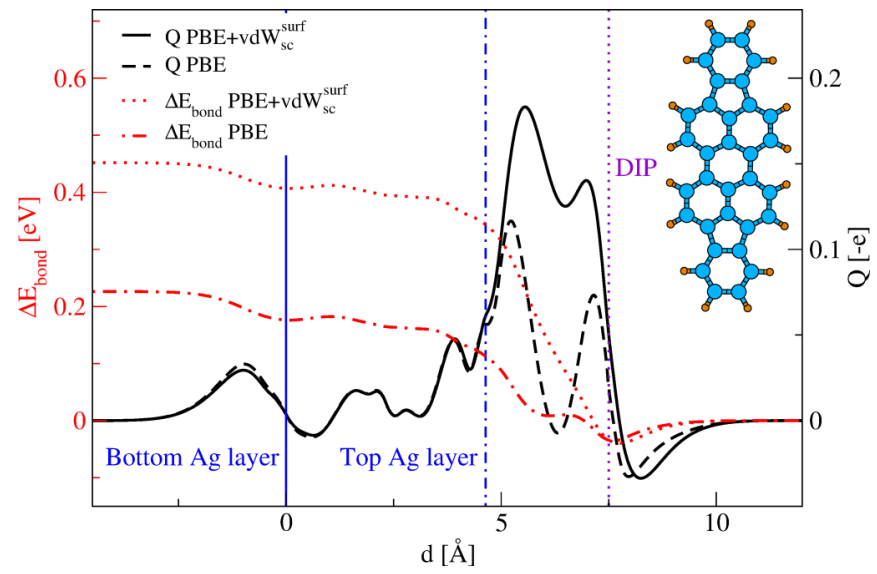

FIG. 3. DIP on $\operatorname{Ag}(111)$. Charge displaced during molecular adsorption $[Q(z)]$ computed with $\mathrm{PBE}+\mathrm{vdW}_{\mathrm{sc}}^{\text {surf }}$ (solid line) and PBE (dashed line). The dotted vertical line refers to the DIP monolayer. The figure shows that the inclusion of vdW effects enhances the accumulation of $Q(z)$ at the interface. This contribution is reflected into a larger change of potential energy $\left(\Delta E_{\text {bond }}\right)$, which ultimately determines the value of the work-function shift. towards the molecule. Here, self-consistency decreases the steepness of PBE $+v_{d W} W^{\text {surf }}$, drastically increasing the value of the local minimum.

The molecular orbital density of states (MODOS) can be employed to compute the occupation of a molecular orbital upon hybridization. Thus, the amount of electrons transferred to the molecule can be quantitatively assessed via a Mulliken-like analysis [57,58]. It results that self-consistency halves the charge transfer, starting from $0.2 e$ with $\mathrm{PBE}$ to $0.09 e$ with $\mathrm{PBE}+\mathrm{vdW}_{\mathrm{sc}}^{\text {surf }}$. In particular, the filling of the lowest unoccupied molecular orbital (LUMO) alone, defined as charge donation, is reduced by as much as $10 \%$. This effect is joined by a quantitatively similar increase of the back donation. In conclusion, from the shape of $Q(z)$ and the MODOS analysis, we obtained conspicuous evidences showing that vdW not only acts upon the pillow effect, but also leads to a large reduction of the charge transfer.

To further establish the role of SC vdW, a simple electrostatic model can be used to partition the work-function shift into two separate contributions:

$$
\Delta \Phi=\Delta E_{\mathrm{mol}}+\mathrm{BD},
$$

where the first term is the contribution coming from the molecular dipole. This term is not connected with the electrostatics at the interface and originates only from the adsorption-induced geometric distortions (bending and stretching phenomena) of the monolayer. In the case of DIP/Ag(111), the relaxed configuration of the molecule is flat, giving a small $\Delta E_{\mathrm{mol}}=$ $-0.042 \mathrm{eV}$. The second term in Eq. (3) is called bond dipole and comes from the formation of dipoles at the interface. The BD is obtained via the solution of the one-dimensional Poisson equation that links $\Delta n(z)$ with the change in the 


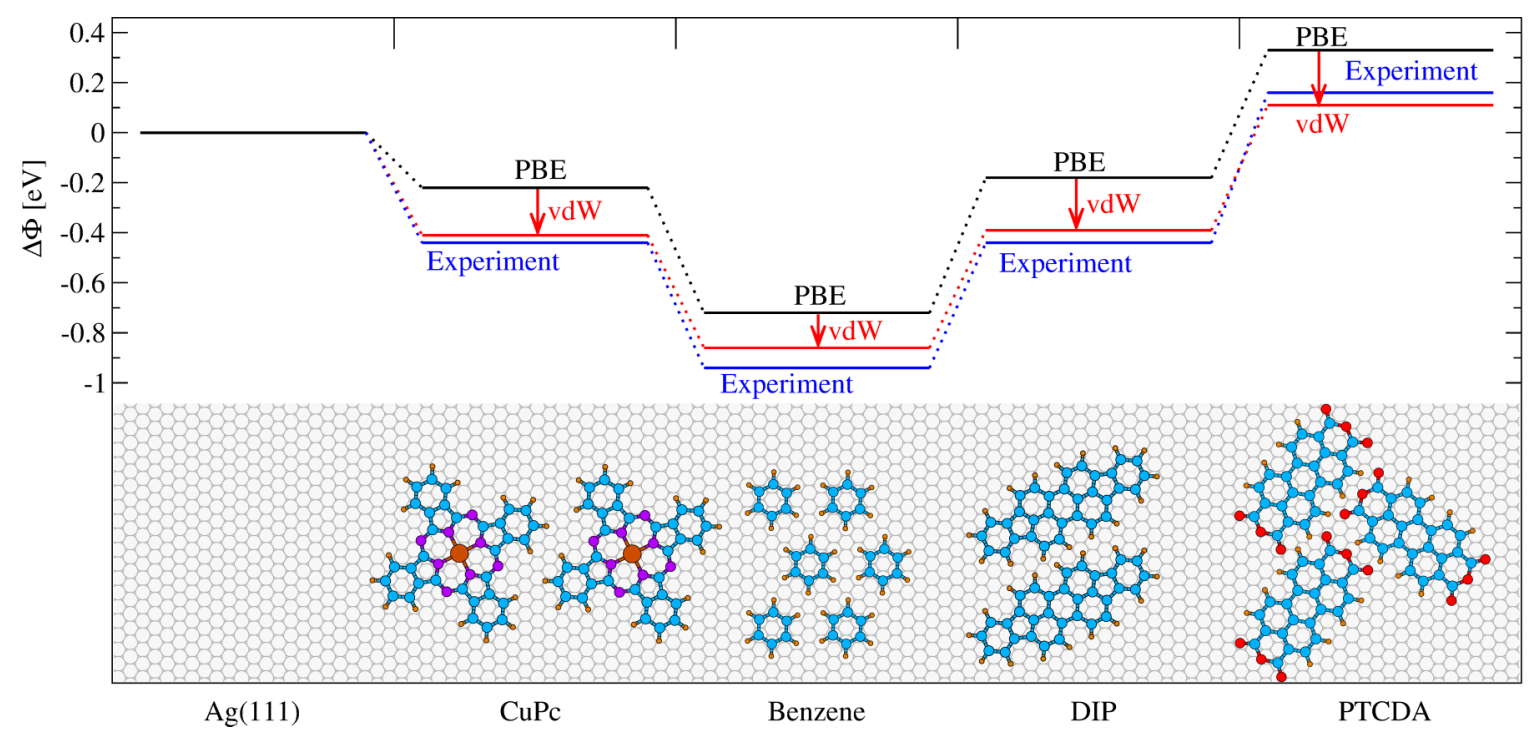

FIG. 4. Adsorption-induced work-function modifications $(\Delta \Phi)$ associated with CuPc, benzene, DIP, and PTCDA on Ag(111). The results of $\mathrm{PBE}$ and $\mathrm{PBE}+\mathrm{vdW}_{\mathrm{sc}}^{\text {surf }}$ are compared with experiments. The reduction of $\Delta \Phi$ due to $\mathrm{SC}$ vdW effects is indicated with an arrow starting from the PBE value. The configurations of the four HMOS are drawn in the bottom half of the figure.

potential energy $\Delta E_{\text {bond }}(z)$ (see Supplemental Material [48]). In Fig. 3 are plotted the $\Delta E_{\text {bond }}(z)$ for DIP $/ \operatorname{Ag}(111)$, computed with $\mathrm{PBE}$ and $\mathrm{PBE}+\mathrm{vdW}_{\mathrm{sc}}^{\text {surf }}$. The $\mathrm{BD}$ corresponds to the fully integrated $\Delta E_{\text {bond }}(z)$, i.e., the constant value on the left side of the figure. The resulting BD are $-0.23 \mathrm{eV}$ for PBE and $-0.45 \mathrm{eV}$ for PBE with SC vdW effects. Notably, the PBE value is doubled with the inclusion of vdW interactions. Finally, the $\Delta \Phi$ obtained with the definition in Eq. (3) is $-0.408 \mathrm{eV}$ for PBE $+\mathrm{vdW}_{\mathrm{sc}}^{\text {surf }}$ and $-0.188 \mathrm{eV}$ for PBE alone, nicely confirming the values reported in Table I.

Our study is concluded with a very well-characterized HMOS: PTCDA on $\operatorname{Ag}(111)[59,60]$. The presence of the functional group $\mathrm{O}=\mathrm{C}-\mathrm{O}-\mathrm{C}=\mathrm{O}$ on both sides of PTCDA enhances the bonding with the metallic surface. This leads to a different behavior with respect to perylenelike molecules. That is, the charge transfer is roughly 3 times larger than DIP/ $\operatorname{Ag}(111)$. The large charge transfer overturns the pillow effect and leads to an increase of the pristine metal work function upon molecular adsorption, i.e., a positive $\Delta \Phi$ [13]. As seen with DIP $/ \operatorname{Ag}(111)$, the inclusion of vdW effects damps the charge transfer and, consequently, limits the increase of $\Delta \Phi$. As a result, $\mathrm{PBE}+\mathrm{vdW}_{\mathrm{sc}}^{\text {surf }}$ applied to PTCDA/ $\mathrm{Ag}(111)$ leads to $\Delta \Phi=+0.11 \mathrm{eV}$, which is one-third of the PBE value and significantly improves the agreement with the experimental data, see Table I. In detail, self-consistency yields a larger Pauli pushback, which is combined with a reduction of about $16 \%$ ( 0.1 e per molecule) in the transfer of charge. In addition to that, around $6 \%$ of the charge donation is redirected as back donation. We consider now the definition of $\Delta \Phi$ in Eq. (3). The value of $\mathrm{BD}$ is $0.48 \mathrm{eV}$ for $\mathrm{PBE}$ and $0.25 \mathrm{eV}$ for $\mathrm{PBE}+\mathrm{vdW}_{\mathrm{sc}}^{\text {surf }}$. The molecular dipole is $-0.17 \mathrm{eV}$ for both functionals. We note in passing that the changes in the PTCDA geometry during adsorption produce a $\Delta E_{\mathrm{Mol}}$ roughly 4 times larger than that of DIP. The resulting $\Delta \Phi$ are $0.08 \mathrm{eV}$ and $0.31 \mathrm{eV}$ for PBE with and without SC vdW, respectively, in very good agreement with the results reported in Table I.
All the $\Delta \Phi$ presented in this work are summarized with respect to pristine $\operatorname{Ag}(111)$ in Fig. 4. These findings altogether, compared with the experimental data, provide a clear overview of the fundamental contributions of $\mathrm{SC} \mathrm{vdW}$ in the regulation of the electronic properties of HMOS. In particular, for all the different HMOS considered, the $\Delta \Phi$ computed with vdW effects undergo significant variations with respect to standard PBE calculations. Curiously, self-consistency is always found to improve the agreement with experiments.

\section{CONCLUSIONS}

In our work we studied the role of $\mathrm{vdW}$ interactionsbeyond cohesive effects-in relation to electronic structures and properties of hybrid monolayer/metal systems. This goal is achieved via fully self-consistent calculations. Our findings demonstrate that self-consistency is responsible for a large redistribution of electron density, which affects the electronic properties. In particular, vdW interactions enhance the Pauli pushback effect and reduce the charge transfer. Consequently, large modifications of interface dipoles $(\sim 0.2 \mathrm{D})$ and workfunction shifts (up to $0.22 \mathrm{eV}$ ) are found, leading to a better agreement with experiments. In conclusion, our study brings compelling evidences that the long-range $\mathrm{vdW}$ interactions can be a key factor for predicting and controlling the electronic properties of HMOS. As such, a refined description of the electronic structure of hybrid systems is achieved only with the inclusion of $\mathrm{vdW}$ interactions into standard $\mathrm{XC}$ functionals.

\section{ACKNOWLEDGMENTS}

We are grateful for support from the FP7 Marie Curie Actions of the European Commission via the Initial Training Network SMALL (MCITN-238804) and the Collaborative Research Center (CRC) 951 of the German Science Foundation. 
[1] N. Koch, ChemPhysChem 8, 1438 (2007).

[2] L. Kronik and N. Koch, MRS Bull. 35, 417 (2010).

[3] N. Nerngchamnong, L. Yuan, D.-C. Qi, J. Li, D. Thompson, and C. A. Nijhuis, Nat. Nanotechnol. 8, 113 (2013).

[4] W. Liu, S. N. Filimonov, J. Carrasco, and A. Tkatchenko, Nat. Commun. 4, 2569 (2013).

[5] H. Ishii, K. Sugiyama, E. Ito, and K. Seki, Adv. Mater. 11, 605 (1999).

[6] H. Ishii, K. Sugiyama, D. Yoshimura, E. Ito, Y. Ouchi, and K. Seki, IEEE J. Sel. Top. Quantum Electron. 4, 24 (1998).

[7] M. Mura, A. Gulans, T. Thonhauser, and L. Kantorovich, Phys. Chem. Chem. Phys. 12, 4759 (2010).

[8] O. T. Hofmann, J.-C. Deinert, Y. Xu, P. Rinke, J. Stähler, M. Wolf, and M. Scheffler, J. Chem. Phys. 139, 174701 (2013).

[9] W. Liu, V. G. Ruiz, G.-X. Zhang, B. Santra, X. Ren, M. Scheffler, and A. Tkatchenko, New J. Phys. 15, 053046 (2013).

[10] W. Liu, J. Carrasco, B. Santra, A. Michaelides, M. Scheffler, and A. Tkatchenko, Phys. Rev. B 86, 245405 (2012).

[11] C. Bürker, N. Ferri, A. Tkatchenko, A. Gerlach, J. Niederhausen, T. Hosokai, S. Duhm, J. Zegenhagen, N. Koch, and F. Schreiber, Phys. Rev. B 87, 165443 (2013).

[12] V. G. Ruiz, W. Liu, E. Zojer, M. Scheffler, and A. Tkatchenko, Phys. Rev. Lett. 108, 146103 (2012).

[13] L. Romaner, D. Nabok, P. Puschnig, E. Zojer, and C. AmbroschDraxl, New J. Phys. 11, 053010 (2009).

[14] Y. L. Huang, E. Wruss, D. A. Egger, S. Kera, N. Ueno, W. A. Saidi, T. Bucko, A. T. S. Wee, and E. Zojer, Molecules 19, 2969 (2014).

[15] N. Ferri, R. A. DiStasio, A. Ambrosetti, R. Car, and A. Tkatchenko, Phys. Rev. Lett. 114, 176802 (2015).

[16] G. Heimel, L. Romaner, E. Zojer, and J.-L. Brédas, Nano Lett. 7, 932 (2007).

[17] O. T. Hofmann, D. A. Egger, and E. Zojer, Nano Lett. 10, 4369 (2010).

[18] O. T. Hofmann, V. Atalla, N. Moll, P. Rinke, and M. Scheffler, New J. Phys. 15, 123028 (2013).

[19] W. Liu, A. Tkatchenko, and M. Scheffler, Acc. Chem. Res. 47, 3369 (2014).

[20] S. Braun, M. P. de Jong, W. Osikowicz, and W. R. Salaneck, Appl. Phys. Lett. 91, 202108 (2007).

[21] F. Tautz, Prog. Surf. Sci. 82, 479 (2007).

[22] A. Crispin, X. Crispin, M. Fahlman, M. Berggren, and W. R. Salaneck, Appl. Phys. Lett. 89, 213503 (2006).

[23] S. Braun, W. R. Salaneck, and M. Fahlman, Adv. Mater. 21, 1450 (2009).

[24] P. C. Rusu, G. Giovannetti, C. Weijtens, R. Coehoorn, and G. Brocks, J. Phys. Chem. C 113, 9974 (2009).

[25] H. H. Kim, J. W. Yang, S. B. Jo, B. Kang, S. K. Lee, H. Bong, G. Lee, K. S. Kim, and K. Cho, ACS Nano 7, 1155 (2013).

[26] B. Kang, S. Lim, W. H. Lee, S. B. Jo, and K. Cho, Adv. Mater. 25, 5856 (2013)

[27] W. Gao and A. Kahn, Appl. Phys. Lett. 79, 4040 (2001).

[28] X. Crispin, V. Geskin, A. Crispin, J. Cornil, R. Lazzaroni, W. R. Salaneck, and J.-L. Brédas, J. Am. Chem. Soc. 124, 8131 (2002).
[29] N. Koch, S. Duhm, J. P. Rabe, A. Vollmer, and R. L. Johnson, Phys. Rev. Lett. 95, 237601 (2005).

[30] E. Goiri, M. Matena, A. El-Sayed, J. Lobo-Checa, P. Borghetti, C. Rogero, B. Detlefs, J. Duvernay, J. E. Ortega, and D. G. de Oteyza, Phys. Rev. Lett. 112, 117602 (2014).

[31] Z. Ma, F. Rissner, L. Wang, G. Heimel, Q. Li, Z. Shuai, and E. Zojer, Phys. Chem. Chem. Phys. 13, 9747 (2011).

[32] C. Hückstädt, S. Schmidt, S. Hüfner, F. Forster, F. Reinert, and M. Springborg, Phys. Rev. B 73, 075409 (2006).

[33] S. Grimme, Wiley Interdiscip. Rev. Comput. Mol. Sci. 1, 211 (2011).

[34] A. Tkatchenko and M. Scheffler, Phys. Rev. Lett. 102, 073005 (2009).

[35] H. Rydberg, M. Dion, N. Jacobson, E. Schröder, P. Hyldgaard, S. I. Simak, D. C. Langreth, and B. I. Lundqvist, Phys. Rev. Lett. 91, 126402 (2003)

[36] M. Dion, H. Rydberg, E. Schröder, D. C. Langreth, and B. I. Lundqvist, Phys. Rev. Lett. 92, 246401 (2004).

[37] V. Blum, R. Gehrke, F. Hanke, P. Havu, V. Havu, X. Ren, K. Reuter, and M. Scheffler, Comput. Phys. Commun. 180, 2175 (2009).

[38] J. P. Perdew, K. Burke, and M. Ernzerhof, Phys. Rev. Lett. 77, 3865 (1996).

[39] I. Kröger, B. Stadtmüller, C. Stadler, J. Ziroff, M. Kochler, A. Stahl, F. Pollinger, T.-L. Lee, J. Zegenhagen, F. Reinert, and C. Kumpf, New J. Phys. 12, 083038 (2010).

[40] W. Liu, F. Maaß, M. Willenbockel, C. Bronner, M. Schulze, S. Soubatch, F. S. Tautz, P. Tegeder, and A. Tkatchenko, Phys. Rev. Lett. 115, 036104 (2015).

[41] C. T. Campbell and J. R. V. Sellers, J. Am. Chem. Soc. 134, 18109 (2012).

[42] X.-L. Zhou, M. Castro, and J. White, Surf. Sci. 238, 215 (1990).

[43] T. Hosokai (private communication).

[44] A. Hauschild, R. Temirov, S. Soubatch, O. Bauer, A. Schöll, B. C. C. Cowie, T.-L. Lee, F. S. Tautz, and M. Sokolowski, Phys. Rev. B 81, 125432 (2010).

[45] S. Duhm, A. Gerlach, I. Salzmann, B. Bröker, R. Johnson, F. Schreiber, and N. Koch, Org. Electron. 9, 111 (2008).

[46] Y.Zou, L. Kilian, A. Schöll, T. Schmidt, R. Fink, and E. Umbach, Surf. Sci. 600, 1240 (2006).

[47] I. Kröger, B. Stadtmüller, C. Kleimann, P. Rajput, and C. Kumpf, Phys. Rev. B 83, 195414 (2011).

[48] See Supplemental Material at http://link.aps.org/supplemental/ 10.1103/PhysRevMaterials.1.026003 for specific settings and details of the calculations.

[49] P. Yannoulis, R. Dudde, K. H. Frank, and E. E. Koch, Surf. Sci. 189-190, 519 (1987).

[50] R. Dudde, K. H. Frank, and E. E. Koch, Surf. Sci. 225, 267 (1990).

[51] T. S. Chwee and M. B. Sullivan, J. Chem. Phys. 137, 134703 (2012).

[52] A. K. Tripathi and J. Pflaum, Appl. Phys. Lett. 89, 082103 (2006).

[53] A. C. Dürr, F. Schreiber, M. Münch, N. Karl, B. Krause, V. Kruppa, and H. Dosch, Appl. Phys. Lett. 81, 2276 (2002). 
[54] D. G. de Oteyza, E. Barrena, H. Dosch, and Y. Wakayama, Phys. Chem. Chem. Phys. 11, 8741 (2009).

[55] H. Huang, J.-T. Sun, Y. P. Feng, W. Chen, and A. T. S. Wee, Phys. Chem. Chem. Phys. 13, 20933 (2011).

[56] D. G. de Oteyza, E. Barrena, M. Ruiz-Osés, I. Silanes, B. P. Doyle, J. E. Ortega, A. Arnau, H. Dosch, and Y. Wakayama, J. Phys. Chem. C 112, 7168 (2008).

[57] R. S. Mulliken, J. Chem. Phys. 36, 3428 (1962).
[58] L. Romaner, Modelling of Organic Semiconductors and Their Interaction With Organic Surfaces (Graz University of Technology, Graz, Austria, 2007).

[59] K. Glöckler, C. Seidel, A. Soukopp, M. Sokolowski, E. Umbach, M. Böhringer, R. Berndt, and W.-D. Schneider, Surf. Sci. 405, 1 (1998).

[60] A. Kraft, R. Temirov, S. K. M. Henze, S. Soubatch, M. Rohlfing, and F. S. Tautz, Phys. Rev. B 74, 041402 (2006). 